\title{
Expansión a largo plazo de la capacidad de Generación: caso del mercado acoplado Perú-Chile
}

\author{
Fabiola Jara, Jaime E. Luyo, José Meza \\ Programa de Doctorado en Ciencias con Mención en Energética, Universidad Nacional de Ingeniería, Av. \\ Tupac Amaru 210, Rímac, Lima, Perú
}

Enviado el 21 de diciembre del 2021. Aceptado el 9 de enero 2022.

DOI: https://doi.org/10.33017/RevECIPeru2021.0011/

\section{Resumen}

La tendencia internacional y en la región sudamericana hacia formación de un mercado único de electricidad mediante la interconexión progresiva de mercados de países vecinos es un reto actual ya que se deben identificar, comprender y resolver los problemas de las diferentes políticas energéticas adoptadas en los países. Sin embargo, cada país ha venido aplicando unilateralmente políticas diferenciadas de desarrollo energético y suficiencia de generación que han influenciado el comportamiento de su mercado eléctrico interno. En el presente artículo se investiga los efectos de la aplicación de estas políticas unilaterales y aisladas de desarrollo energético, de subsidios a los combustibles, de la variación del precio de los combustibles, de suficiencia de oferta de capacidad de generación en base de fuentes renovables, no-renovables y de nueva tecnología; así como de la ampliación del interconector. Para ello, se ha estructurado un modelo que hace uso de la teoría de dinámica de sistemas y se analizó el caso de estudio de la interconexión Perú - Chile, se concluyó que la diferencia que existe en la disponibilidad de recursos energéticos NR (gas natural) entre Perú y Chile ha influido en diferencia entre los costos marginales de sus sistemas eléctricos (CMg9, que en todos los escenarios simulados la mayor parte del intercambio de energía se realiza desde Perú a Chile representando entre el $98 \%$ y el $79 \%$ del total de intercambios durante todo el periodo. también, para el caso de ausencia del gasoducto sur peruano, se alcanza la suficiencia de capacidad eléctrica en el Perú, No se observa en el corto ni en el mediano plazo la existencia de complementariedad energética entre ambos países. Ambos países pueden llegar tener una matriz eléctrica de oferta del 75\% RER al 2050.

Descriptores: Interconexión, mercado eléctrico, política regulatoria, simulación, complementariedad hídrica

\section{Abstract}

The international trend and in the South American region towards the formation of a single electricity market through the progressive interconnection of markets of neighboring countries is a current challenge since the problems of the different energy policies adopted in the countries must be identified, understood and solved. However, each country has unilaterally applied differentiated energy development and generation sufficiency policies that have influenced the behaviour of its internal electricity market. This article investigates the effects of the application of these unilateral and isolated policies of energy development, fuel subsidies, the variation in the price of fuels, the sufficiency of supply of generation capacity based on renewable sources, non-renewable and new technology, as well as the expansion of the interconnector. For this, a model that makes use of the system dynamics theory has been structured and the case study of the Peru - Chile interconnection was analysed, it was concluded that the difference that exists in the availability of energy resources NR (natural gas) between Peru and Chile has influenced the difference between the marginal costs of their electrical systems (CMg9, that in all simulated scenarios most of the energy exchange is carried out from Peru to Chile, representing between $98 \%$ and $79 \%$ of the total exchanges throughout the period. Also, in the case of the absence of the southern Peruvian gas pipeline, the sufficiency of electrical capacity is reached in Peru, the existence of energy complementarity between both countries is not observed in the short or medium term. Countries may have a $75 \%$ RER electricity supply matrix by 2050 .

Keywords: Interconnection, electricity market, regulation policies, simulation, water complementarity 


\section{Introducción}

Desde el inicio de la presente centuria se está intensificando los procesos de integración energética regionales $y$, particularmente en la región latinoamericana la integración de mercados eléctricos a través de las líneas de interconexión eléctrica entre los países vecinos, que permiten optimizar las inversiones ampliando los mercados, por mayor seguridad energética sobre todo de países que no son autosuficientes y, para lograr la sostenibilidad energética a largo plazo [1], para que la integración progrese, aparte de la interconexión física se tienen que superar algunos retos, debido a que, es muy difícil llegar a acuerdos porque las políticas internas de regulación que rigen en los países vecinos muchas veces obstaculizan el funcionamiento de estos acuerdos, otro de los factores es la diferencia entre los tipos de mercados de electricidad; sin embargo, la interconexión puede servir también para mejorar la organización y diseño de dichos mercados [2] y [3]. En general, se producen problemas regulatorios y de coordinación en la integración de los mercados de países fronterizos por factores técnicos, políticos y económicos diferenciados en los mercados eléctricos en cada país [4], y particularmente por las políticas energéticas adoptadas en los países, como se refiere a continuación.

Las interconexiones son en general convenientes, ya que los recursos energéticos que poseen los países no son igualmente distribuidos, además pueden contribuir a reducir los precios de energía e incrementar el suministro en regiones con mayor demanda, aumentando los ingresos del país exportador de los costos de suministro a largo plazo [5].

El objetivo de la interconexión transfronteriza de electricidad es principalmente la optimización en el uso de recursos lo cual conlleva una reducción de costos de generación; sin embargo, los mecanismos de capacidad generan inquietudes acerca de la confiabilidad, debido a que las rentas de congestión de la interconexión se dividen entre ambos países, y esto puede originar que las inversiones sean difíciles asumir para un generador exportador en el país exportador ya que estaría asumiendo una obligación que está fuera de su control en el país importador [6].

Estos eventos afectan la eficiencia de la interconexión a corto y a largo plazo por eso es importante estudiarlos mediante diferentes escenarios, teniendo en cuenta: i) los mecanismos de capacidad, ii) la participación en el mercado interconectado y iii) características propias del mercado nacional.

El objetivo de la presente investigación es mejorar la comprensión de los efectos que tendrá la interconexión de mercados eléctricos transfronterizos en los siguientes puntos:

- La evolución del parque de generación electricidad en cada sistema eléctrico.

- Identificar si hay complementariedad energética, Perú con una política de desarrollo de generación termoeléctrica a gas natural y Chile hacia la implementación de plantas de generación con fuentes renovables no convencionales.

- La incorporación de centrales eléctricas a base de recursos energéticos renovables no convencionales y nueva tecnología y, la ampliación de la capacidad del interconector.

- El impacto en los costos marginales en Perú y Chile con la interconexión eléctrica

Para alcanzar dicho objetivo se ha mejorado el modelo propuesto por Jara et al [7] y presentado en [8], el cual hace uso de la teoría de dinámica de sistemas para interrelacionar:

i. Un modelo de expansión de la generación con un horizonte de simulación de largo plazo el cual sea suficiente para observar la evolución de las diferentes tecnologías de generación y cuenta con una resolución mensual que permita observar las variaciones estacionales de la oferta debidas a la hidrología.

ii. Un modelo de despacho económico de corto plazo para determinar el impacto en los costos marginales que afectarán la rentabilidad de los nuevos proyectos de generación, así como de las líneas de interconexión.

El análisis realizado se centra en el caso particular de Perú y Chile, en la sección 2 se hace una descripción del modelo empleado, en la sección 3 se presentan los resultados del análisis realizado para el caso de la interconexión entre Perú y Chile; Finalmente, en sección 4 se presentan las conclusiones. 


\section{Metodología}

Para analizar el efecto de la interconexión de dos sistemas eléctricos se desarrolló un modelo que consideró el desarrollo dinámico del parque de generación y transmisión, Ver Figura 1.

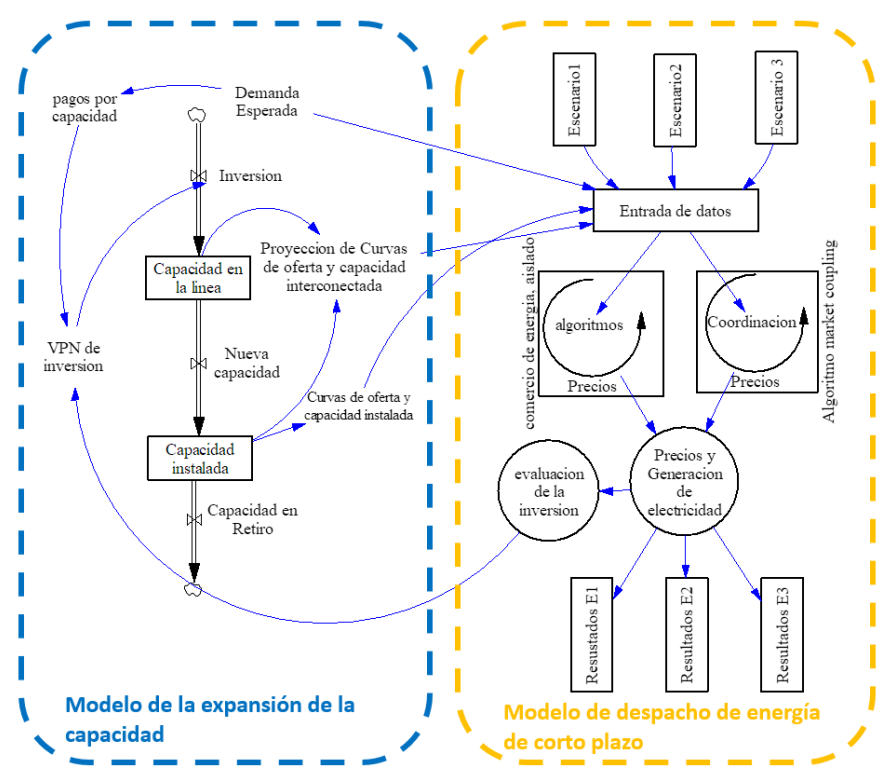

Figura 1. Metodología Propuesta

El modelo de mercados de electricidad transfronterizos basado en la dinámica de sistemas está expresado mediante el diagrama de causalidad que se muestra en la Figura 2.

El modelo propuesto consta de dos submodelos: un modelo para el despacho de energía de corto plazo y evaluación de inversiones y un modelo de expansión de la capacidad del sistema de largo plazo, los cuales interactúan y se retroalimentan de manera permanente.

La simulación comienza con el modelo de despacho, que proporciona la cantidad y costos marginales de la energía suministrada por cada planta de generación para cubrir la demanda en cada uno de los países; así como, si los países están interconectados, la energía importada/exportada y las rentas de congestión. A continuación, dicha información es ingresada al modelo de expansión de capacidad y es el punto de partida para la simulación en dicho modelo, el cual sobre la base de indicadores de rentabilidad calcula la incorporación de nuevas plantas de generación por tipo de tecnología; así como, la expansión o no de la línea de interconexión. Finalmente, dicha información es retroalimentada al modelo de despacho para que inicialice nuevamente los cálculos para el siguiente periodo evaluado.

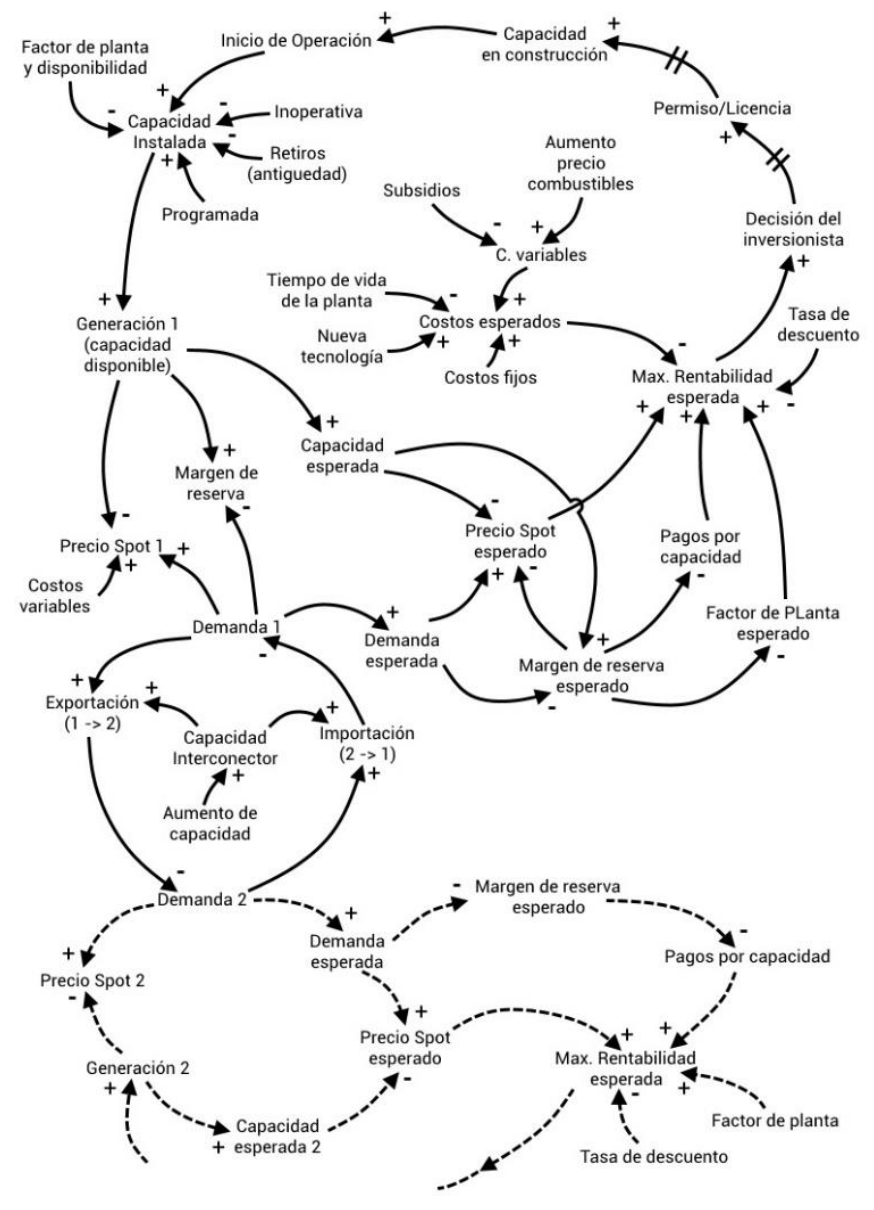

Figura 2. Diagrama de causalidad del modelo de mercados de electricidad transfronterizos

\subsection{Modelo de despacho y cálculo del VPN}

El modelo se basa en un algoritmo de despacho por orden de mérito de corto plazo que simula el despacho de energía por hora, tanto para su operación en forma aislada, con interconexión y con interconexión ilimitada, para ello toma en cuenta los principios de acoplamiento de mercado y desarrolla los siguientes procesos:

- Calcula la generación por fuente de cada país, en términos de energía eléctrica generada y costos marginales, flujos por las líneas de interconexión y rentas de congestión.

- Evalúa las inversiones, y calcula el valor presente neto (VPN) de las inversiones por cada tecnología.

El modelo de programación del despacho económico se formula de acuerdo con las ecuaciones (1) y (2). 
$\min \sum_{t}\left[\sum_{j} C V_{j}\left(G_{j}(t)\right)+C A(V(t+1))\right]$

$\sum_{j} G_{j}(t)+G_{h}(t)=D(t) \pm F I(t)-G_{S}(t)-$

$G_{e}(t)-G_{S C}(t)$

Sujeto a:

$|F I(t)| \leq F I \max$

$V \min \leq V(t)<V \max$

$G_{j} \min \leq G_{j}(t)<G_{j} \min$

Donde:

$C V_{i}$ : Costo variable de tecnologías de generación (no incluye Hidro, Eólico y solar)

CA : Costo de oportunidad del agua (generación Hidro con embalse)

$\mathrm{V}(\mathrm{t}) \quad$ : Volumen total embalse al final del periodo $\mathrm{t}$

$\mathrm{FI}(\mathrm{t})$ : Flujo en el Interconector en el periodo $\mathrm{t}$

$G_{j}(t)$ : Generación termoeléctrica en el periodo $t$

$\mathrm{D}(\mathrm{t}) \quad$ : Demanda en el periodo $\mathrm{t}$

$G_{h}(t)$ : Generación hidroeléctrica en el periodo $t$

$G_{S}(t)$ : Generación solar fotovoltaica en el periodo $\mathrm{t}$

$G_{e}(t)$ : Generación eólica en el periodo $t$

$G_{S C}(t)$ : Generación solar de concentración en el periodo $t$

La solución óptima a este problema es equivalente a ordenar los generadores de menor a mayor costo variable en una lista de mérito, el operador del sistema despacha las unidades en ese orden hasta cubrir la demanda en cada período. Este algoritmo fue implementado en MATLAB.

\subsection{Modelo de Expansión}

El modelo de expansión simula la evolución del parque de generación, así como la disponibilidad que tendrán durante su operación. Para ello se han estructurado 3 módulos los cuales se listan a continuación:

a) Módulo de evaluación de la inversión

Se realiza la evaluación de la inversión de las tecnologías disponibles, para ello se tiene en cuenta el retardo por el proceso de obtención de permisos y la construcción hasta la puesta en operación de la central.

b) Módulo del ciclo de vida de la capacidad instalada

Se evalúa el ciclo de vida del parque de generación y la línea de interconexión, desde el momento de la inversión hasta el momento del retiro de la unidad, La capacidad instalada determina las curvas de oferta y la capacidad de la línea de interconexión, que son los datos que se exportan al modelo de despacho para calcular las cantidades de energía generada y costos marginales. Este módulo también evalúa un análisis de esquemas de pagos por capacidad que podrían ser implementados en los escenarios evaluados.

\section{Resultados y Discusión de Resultados}

A fin de investigar el efecto de las políticas unilaterales orientadas a la incorporación de la tecnología de generación con fuente energética no renovable como el caso del GN en el Perú y de fuentes renovables en Chile, como países fronterizos con mercados acoplados. Se considera el efecto de la ampliación del enlace (interconector) asumiendo que ha sido concordado por ambos países, el pago por capacidad que ambos países están aplicando se mantiene $\mathrm{y}$, la variación del precio del GN a lo largo del tiempo. Además, para tomar en cuenta el posible intercambio energético Perú-Chile, el perfil de la demanda eléctrica peruana es proyectada internamente y se considera con una tasa de crecimiento conservadora. El periodo de simulación es 2019-2050.

Los escenarios estudiados permiten comprender mejor el comportamiento dinámico de estos mercados transfronterizos y el efecto de políticas energéticas unilaterales, que se reflejan en la variación del precio de la electricidad, la matriz eléctrica de oferta, en el corto y largo plazo y, sobre todo comprobar si hay complementariedad energética en los mercados acoplados de los dos países.

A fin de evaluar las diferentes opciones, se han simulado seis escenarios, los cuales se listan en la Tabla I.

El escenario PC01 es el escenario base de la interconexión, donde se considera que la interconexión de $200 \mathrm{MW}$ empieza a operar el año 2023 y en el 2032 operaría una ampliación del interconector de 500MW adicionales, que el precio del GN es variable en ambos países y varía según se muestra en la Tabla II, también ambos sistemas contarán con nueva tecnología termo solar de concentración (CSP, en inglés); también, se considera la operando del gasoducto sur peruano desde el 2026 y que Chile alcanza su meta de $70 \%$ de participación de recursos energéticos renovables (RER) [9]; los otros escenarios son sensibilidades de este escenario.

El escenario PC02 evalúan el efecto de mantener el precio de GN en Perú constante, lo cual implicaría que se continuaría con el esquema actual de 
subsidio. El escenario PC03 evalúa el efecto de no implementar GSP en el año 2026, mientras que el escenario PC04 evalúa el efecto de no cumplir con la meta de Chile de tener como mínimo el $70 \%$ de la matriz eléctrica de oferta con centrales RER en el 2050. Por su parte el escenario PC05 evalúa el efecto de no implementar centrales CSP. Finalmente, el escenario PC06 evalúa el desenvolvimiento que tendrían los sistemas si no se llevara a cabo la interconexión.

Tabla 1: Escenarios evaluados

\begin{tabular}{|c|c|c|c|c|c|}
\hline \\
\hline Escenario & Interconexión & Precio de GN & CSP & GSP & RER $>70 \%$ \\
\hline PC01 & \multirow{5}{*}{$\begin{array}{l}\text { 2023: } 200 \mathrm{MW} \\
\text { 2032: } 500 \mathrm{MW}\end{array}$} & $\begin{array}{l}\mathrm{P}: \text { Variable } \\
\mathrm{C}: \text { Variable }\end{array}$ & $\mathrm{Si}$ & $\mathrm{Si}$ & Si \\
\hline PC02 & & $\begin{array}{l}\text { P: Constante } \\
\text { C: Variable }\end{array}$ & $\mathrm{Si}$ & $\mathrm{Si}$ & $\mathrm{Si}$ \\
\hline PC03 & & \multirow{4}{*}{$\begin{array}{l}\text { P: Variable } \\
\text { C: Variable }\end{array}$} & $\mathrm{Si}$ & No & $\mathrm{Si}$ \\
\hline PC04 & & & $\mathrm{Si}$ & $\mathrm{Si}$ & No \\
\hline PC05 & & & No & $\mathrm{Si}$ & Si \\
\hline PC06 & No & & $\mathrm{Si}$ & $\mathrm{Si}$ & $\mathrm{Si}$ \\
\hline
\end{tabular}

Nota: P: Perú, C: Chile

Tabla 2: Escenarios de precio del GN generación (US\$MMBTU)

\begin{tabular}{|l|l|l|l|l|l|l|l|l|l|l|l|l|}
\hline País & Escenario & 2018 & 2019 & 2020 & 2021 & 2022 & 2023 & 2024 & 2025 & 2026 & 2027 & 2028 \\
\hline \multirow{2}{*}{ Perú } & Constante & 0.69 & 0.69 & 0.69 & 3.10 & 3.10 & 3.10 & 3.10 & 3.10 & 3.10 & 3.10 & 3.10 \\
\cline { 2 - 14 } & Variable & 0.69 & 0.69 & 0.69 & 3.10 & 3.10 & 5.30 & 5.30 & 5.80 & 5.80 & 5.80 & 5.80 \\
\hline Chile & Variable & 8.25 & 8.25 & 8.25 & 8.15 & 8.16 & 8.31 & 8.51 & 8.78 & 8.87 & 8.90 & 8.99 \\
\hline
\end{tabular}

\begin{tabular}{|l|l|l|l|l|l|l|l|l|l|l|l|l|}
\hline País & Escenario & 2029 & 2030 & 2031 & 2032 & 2033 & 2034 & 2035 & 2036 & 2037 & 2038 & 2039 \\
\hline \multirow{2}{*}{ Perú } & Constante & 3.10 & 3.10 & 3.10 & 3.10 & 3.10 & 3.10 & 3.10 & 3.10 & 3.10 & 3.10 & 3.10 \\
\cline { 2 - 13 } & Variable & 5.80 & 6.80 & 6.80 & 6.80 & 6.80 & 6.80 & 7.30 & 7.30 & 7.30 & 7.30 & 7.30 \\
\hline Chile & Variable & 9.01 & 9.05 & 9.03 & 9.19 & 9.37 & 9.50 & 9.49 & 9.47 & 9.60 & 9.68 & 9.71 \\
\hline
\end{tabular}

\begin{tabular}{|l|l|c|c|c|c|c|c|c|c|c|c|c|}
\hline País & Escenario & 2040 & 2041 & 2042 & 2043 & 2044 & 2045 & 2046 & 2047 & 2048 & 2049 & 2050 \\
\hline Perú & Constante & 3.10 & 3.10 & 3.10 & 3.10 & 3.10 & 3.10 & 3.10 & 3.10 & 3.10 & 3.10 & 3.10 \\
\hline & Variable & 7.80 & 7.80 & 7.80 & 7.80 & 7.80 & 8.30 & 8.30 & 8.30 & 8.30 & 8.30 & 8.30 \\
\hline Chile & Variable & 9.72 & 9.71 & 9.78 & 9.82 & 9.86 & 9.92 & 10.02 & 10.14 & 10.23 & 10.28 & 10.42 \\
\hline
\end{tabular}

A continuación, se describen los principales resultados del escenario PC01 y los resultados para el resto de los escenarios se presentan en el apéndice $B$.

\subsection{Costos Marginales Resultantes}

En el Perú se observa que en el periodo 2018 2020, como se presenta en la Figura 3, el CMg varía entre los 8 y 16 US $\$ / M W h$ en avenida y estiaje; respectivamente, esto debido a la distorsión de precios causada por la declaración del precio casi cero del GN. En el periodo 2021 - 2022 a consecuencia de las modificaciones propuestas para la declaración del precio de GN, en el informe sobre la problemática del régimen de declaración de precios de GN [10], el CMg se incrementa, y pasa a estar entre los 25 y 34 US\$/MWh en época de avenida y estiaje, respectivamente.

En el periodo 2023 - 2030, se observa que el $\mathrm{CMg}$ en época de avenida se incrementa a 42 US $\$ / M W h$ mientras que en época de estiaje se eleva llegando a picos de entre 58 y 54 US\$/MWh, esto debido al incremento en el precio del GN y la puesta en operación de la interconexión en el año 2023; 
posteriormente, el $\mathrm{CMg}$ en estiaje se estabiliza en 50 US $\$ / M W h$ como consecuencia de la puesta en operación del GSP en el año 2026.

Finalmente, en el periodo 2031 - 2050 el CMg de estiaje comienza a incrementarse y llega presentar picos de 62 US\$/MWh y 68 US\$/MWh hacia el final del periodo de evaluación. Asimismo, el $\mathrm{CMg}$ en avenida se incrementa como consecuencia del incremento del precio del GN pasando de los 50 US $\$ / M W h$ a los 52 US $\$ / M W h$. Cabe resaltar que la puesta en operación de centrales CSP, las cuales cuentan con un alto factor de planta (0.73) ayudan a disponer de manera más eficiente de los recursos energéticos, por lo cual el CMg no sufre variaciones significativas en el periodo de análisis. Finalmente, se concluye que el incremento continuo en el $\mathrm{CMg}$ en avenida es consecuencia directa del incremento del precio del GN, ya que estas unidades son las que marginan el sistema.

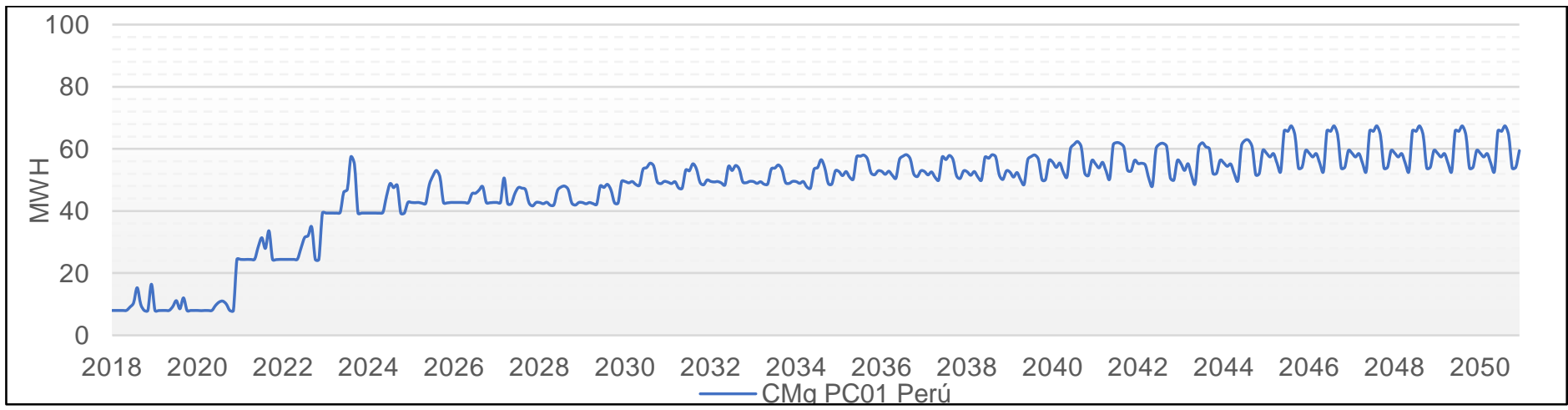

Figura 3: Costos Marginales en Perú para PC01

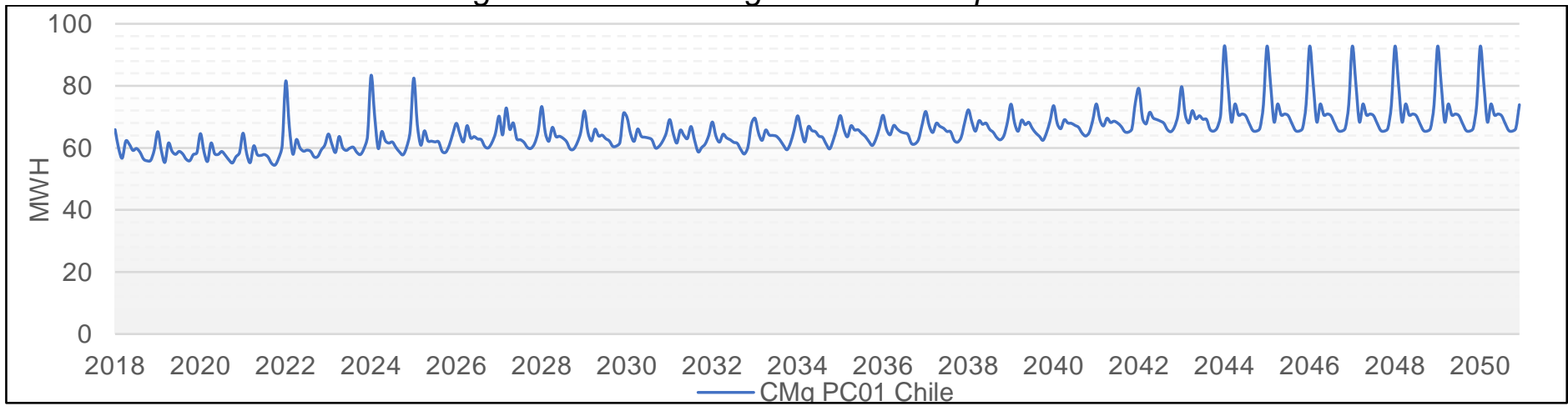

Figura 4: Costos Marginales en Chile para PC01

Los resultados para Chile son presentados en la Figura 4, donde se observa que para el periodo de análisis el CMg de avenida se incrementa pasando de los 58 a los 84 US $\$ / M W h$, esto se debe a que el parque de generación se compone en más del $50 \%$ de centrales térmicas de Carbón, Diesel y GN y aunque las centrales RER aumentan su participación hasta llegar a representa más del $70 \%$ de la matriz de generación de eléctrica en el año 2050 , las centrales térmicas son las que definen el $\mathrm{CMg}$ del sistema.

Por otro lado, el CMg de estiaje presenta picos que llegan a los 70 US $\$ / M W h$ los cuales son atenuados por la posibilidad de importar electricidad desde el Perú a un CMg que es menor al chileno durante todo el periodo de análisis. Para los siete últimos años de análisis el $\mathrm{CMg}$ de estiaje comienza a elevarse hasta llegar a los 92 US\$/MWh lo cual indica que habría la necesidad de ampliar la capacidad del interconector.

\subsection{Flujo de Energía en la Interconexión}

Para visualizar mejor los intercambios de energía entre Perú y Chile se ha dividido el análisis en tres periodos, y se presenta gráficamente la relación entre el CMg y los intercambios de energía, donde se observa que el sentido del intercambio de energía va desde el país que presenta el menor $\mathrm{CMg}$ hacia el que presenta un mayor $\mathrm{CMg}$.

La Figura 5 (a) presenta el periodo 2023 - 2031, donde la capacidad de interconexión es de $200 \mathrm{MW}$, se observa que en este periodo el flujo de Chile a Perú es muy reducido ya que representa solo el $3.2 \%$ del intercambio total (Ver Tabla III), esto se debe al bajo CMg en el Perú con respecto a Chile. 
Asimismo, los meses de julio a octubre son los únicos meses donde se presenta un ligero flujo de cuando durante horas del día hay disponibilidad del Chile hacia Perú, correspondiendo estos a recurso solar en Chile. intercambios en los meses de estiaje en Perú,
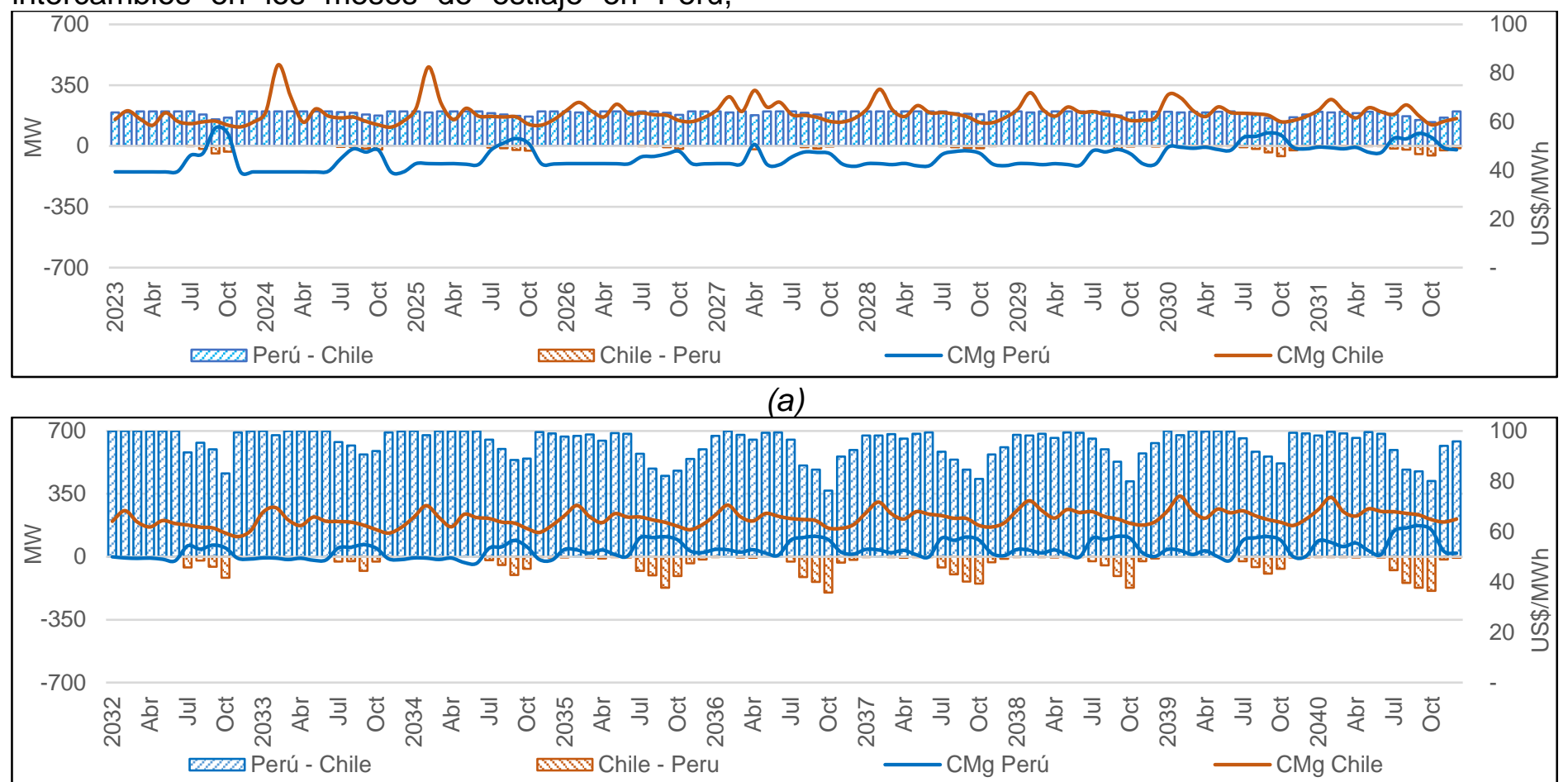

(b)

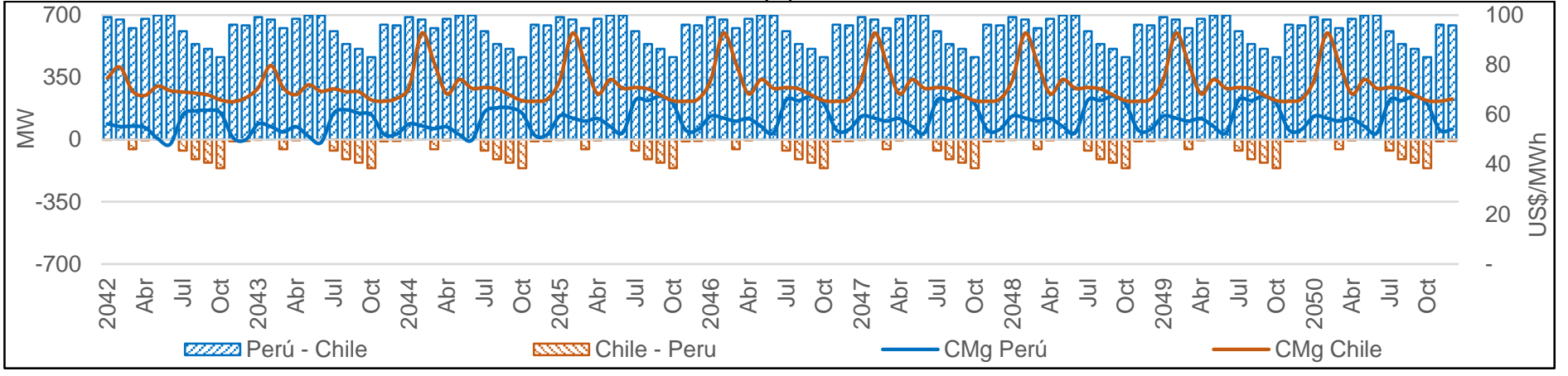

(c)

Figura 5: Flujo de energía en la interconexión en PC01

Para el periodo 2032 - 2041, dado el sinceramiento del precio del GN en el Perú, los CMg de ambos países se acercan y el flujo de chile hacia Perú también aumenta, llegando a representar el 5.2\% del intercambio total (Ver Tabla III), este intercambio se realiza especialmente en los meses de julio a octubre, época de estiaje en Perú, como se presenta en la Figura 5 (b).

Para el periodo 2042 - 2050, dado que el CMg en ambos países es más cercano, y provoca que los intercambios realizados de Chile a Perú se incrementan un poco más, hasta llegar a representar el $6.8 \%$ del intercambio total, esto se realiza en los meses de estiaje en Perú, como se presenta en la Figura 5 (c). Finalmente, a lo largo de todo el periodo de análisis el intercambio de energía desde Chile a Perú solo llega a representa el $5.6 \%$ del intercambio total (Ver Tabla III).

En términos de despacho de electricidad por fuentes, la Figura 6 presenta a manera de ejemplo el despacho realizado para el día 03 de febrero de 2032, época de avenida en Perú, ese día existe flujo exclusivamente desde Perú a Chile durante todo el día, debido a que el $\mathrm{CMg}$ en Perú es inferior al $\mathrm{CMg}$ en Chile todo el tiempo.

Por otro lado, cuando el intercambio es mixto entre ambos países, como es el caso del despacho simulado para el 20 de octubre de 2040 (época de estiaje en Perú), que se presenta en la Figura 7 , 
donde se observa que el intercambio desde chile a Perú se realiza en horas del día cuando el $\mathrm{CMg}$ en Chile es inferior al $\mathrm{CMg}$ del Perú como consecuencia de su alto porcentaje de penetración de energías renovables que para ese año representarían un $62.8 \%$ de la matriz eléctrica de oferta chilena. Mientras que el intercambio desde Perú a Chile se produce cuando el $\mathrm{CMg}$ en Chile sube y supera al CMg de Perú, esto acurre durante las horas en las que no se dispone de recurso solar en Chile. Dentro del escenario evaluado no se presenta el caso donde el intercambio sea exclusivamente desde Chile a Perú durante todo el día.

Tabla 3: Intercambios de energía entre Perú y Chile

\begin{tabular}{|c|c|c|c|}
\hline Periodo & Unidad & Perú - Chile & Chile - Perú \\
\hline \multirow{2}{*}{$2023-2031$} & TWh & 15.16 & 0.51 \\
\cline { 2 - 4 } & $\%$ & 96.8 & 3.2 \\
\hline \multirow{2}{*}{$2032-2041$} & TWh & 55.23 & 3.02 \\
\cline { 2 - 4 } & $\%$ & 94.8 & 5.2 \\
\hline \multirow{2}{*}{$2042-2050$} & TWh & 49.24 & 3.58 \\
\cline { 2 - 4 } & $\%$ & 93.2 & 6.8 \\
\hline \multirow{2}{*}{$2023-2050$} & TWh & 119.63 & 7.10 \\
\cline { 2 - 4 } & $\%$ & 94.4 & 5.6 \\
\hline
\end{tabular}

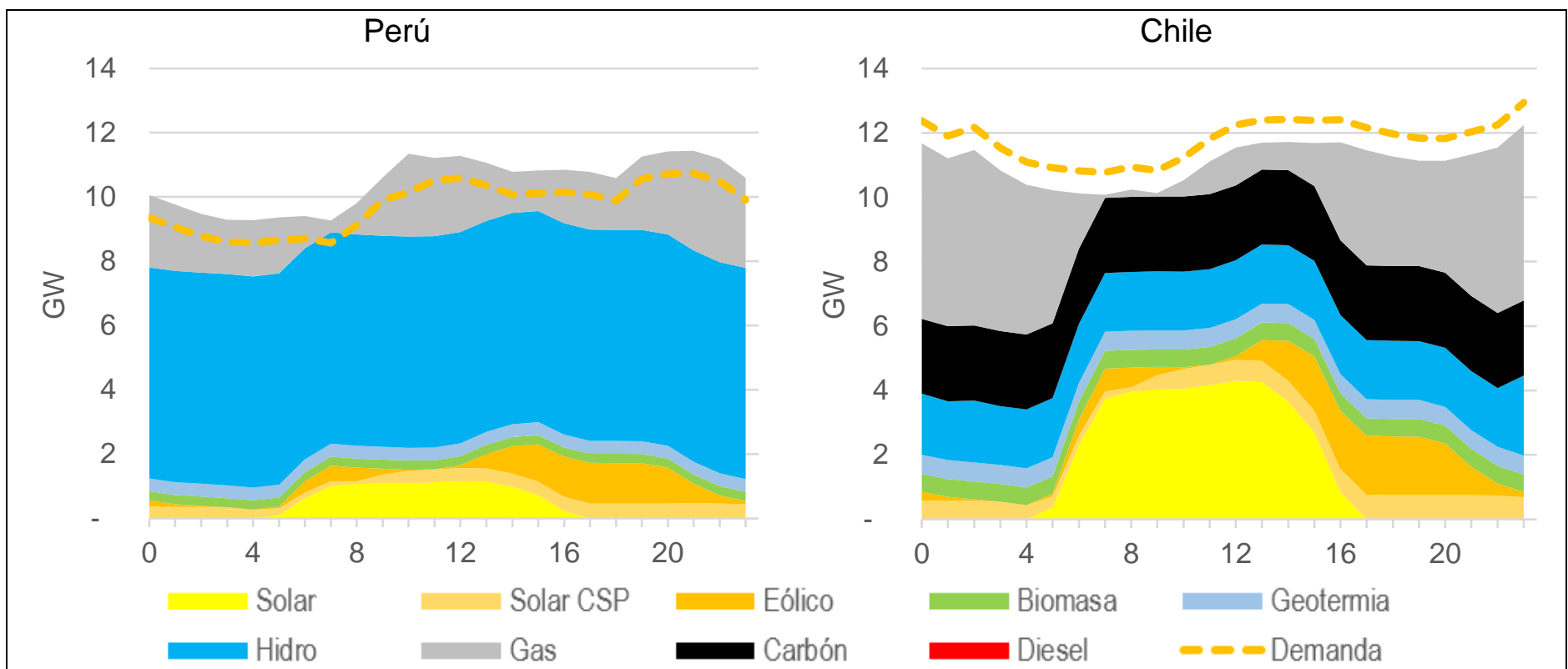

Figura 6: Despacho de electricidad por tipo de tecnología 03/02/32 PC01 (época de avenida en Perú)

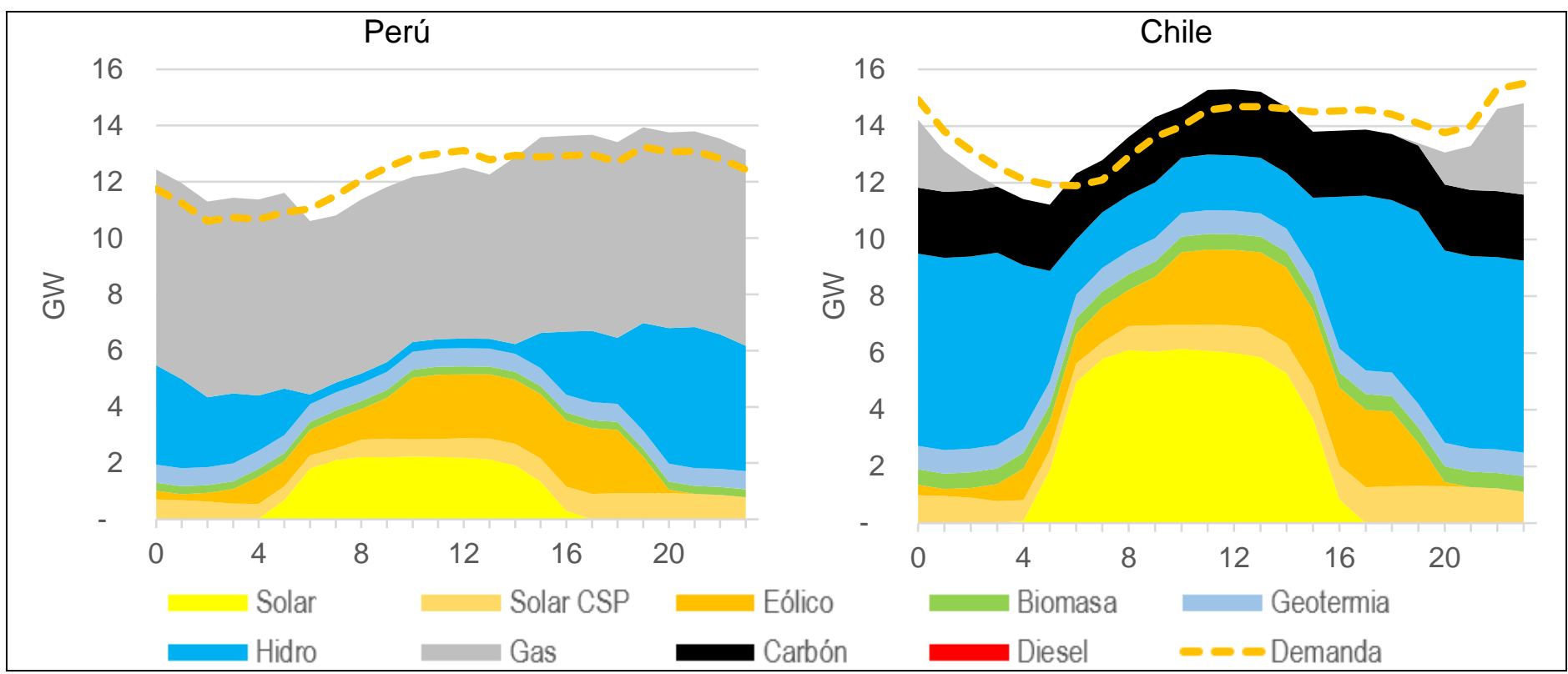

Figura 7: Despacho de electricidad por tipo de tecnología 20/10/40 PC01 (época de estiaje en Perú) 


\subsection{Capacidad Instalada de Generación}

En el escenario PC01, la capacidad instalada de generación en el Perú (sin considerar plantas de reserva fría, pero si el nodo energético de 1,350 MW asociado al GSP) pasa de 11.6 GW en 2019 a 27.7 GW en 2050, mientras que en Chile pasa de $23 \mathrm{GW}$ en 2019 a 44.7 GW en 2050, como se presenta en la Figura 8. Asimismo, se ha considerado el retiro de la única planta a carbón existente en Perú (140 MW) para el año 2023, ya que forma parte de la estrategia global de descarbonización de su propietario [11], en el caso de Chile se considera el plan de retiro de centrales térmicas a carbón de hasta 1661.4 MW [12].

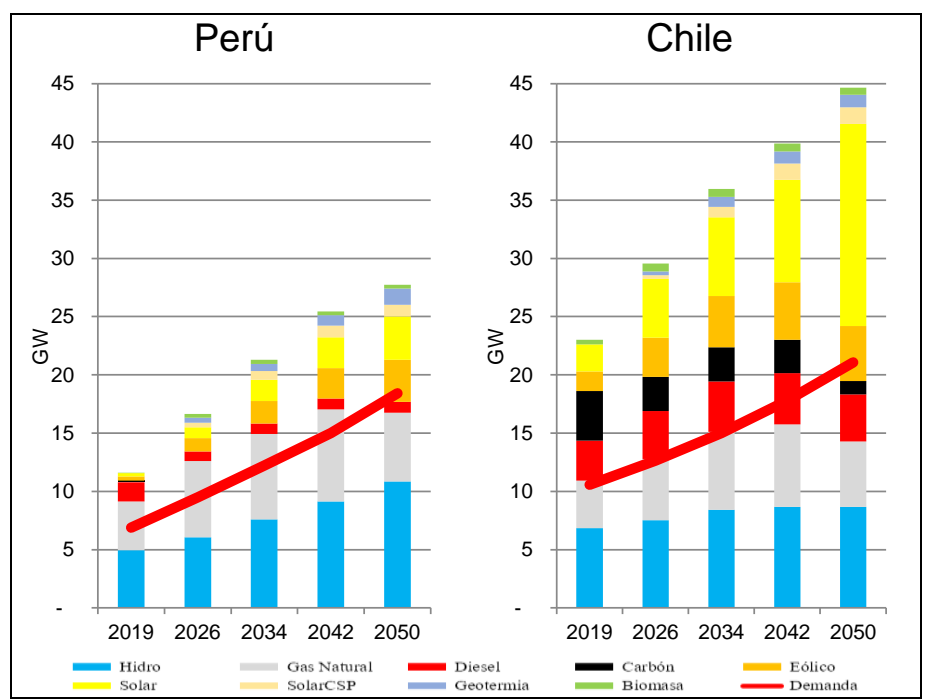

Figura 8: Evolución de la capacidad instalada de generación para PC01

En términos de participación por tecnología, en el Perú se pasa de tener una componente de hidroelectricidad, GN y Diesel de $42.6 \%, 35.9$ y $14.2 \%$ en 2019 a $39.1,21.4 \%$ y $3.2 \%$ en 2050 , respectivamente. Mientras que las energías eólica, solar y biomasa pasan de representar un $3.2 \%$, $2.5 \%$ y $0.3 \%$ en 2019 a $13.1 \%, 13.5 \%$ y $1.2 \%$ en 2050; respectivamente, a lo que se suman las centrales CSP y geotermia, representan el $3.6 \%$ y $5 \%$ respectivamente, como se presenta en la Ver Figura 9.

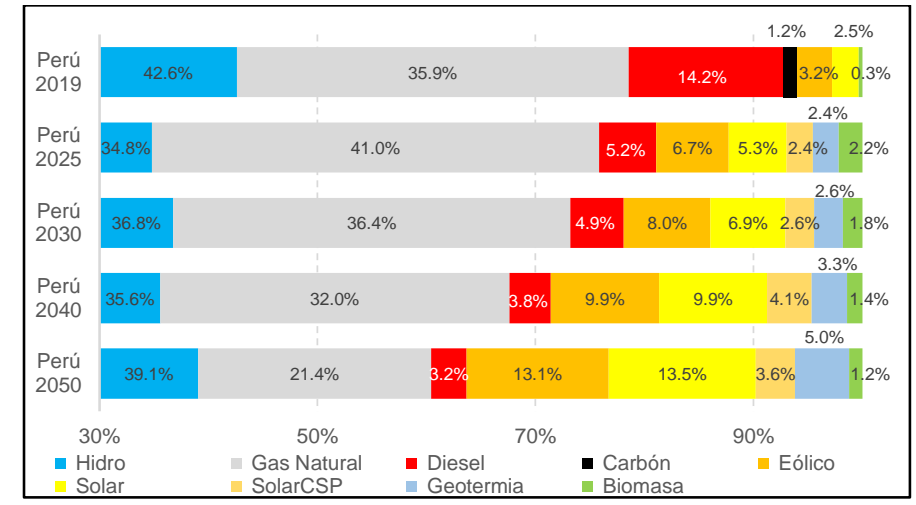

Figura 9: Evolución de la participación por tecnología en Perú para PC01

Por otra parte, en Chile se pasa de tener una componente de hidroelectricidad, GN, Diesel y Carbón de $29.8 \%$, $17.8 \%, 14.9 \%$ y $18.5 \%$ en 2019 a $19.4 \%, 12.6 \%, 9.1 \%$ y $2.5 \%$ en 2050 , respectivamente. Mientras que las energías eólica, solar y biomasa pasan de representar un $7.3 \%$, $10.1 \%$ y $1.6 \%$ en 2018 a $10.6 \%, 38.9 \%$ y $1.4 \%$ en 2050; respectivamente, a lo que se suman las centrales CSP y geotermia, y representan el $3.2 \%$ y $2.3 \%$ en 2050, como se presenta en la Figura 52. Con lo cual \% de participación RER de la matriz eléctrica de oferta chilena totaliza $75.8 \%$ en 2050 y cumple con el lineamiento 4 de la su hoja de ruta 2050 [10].

La variación en la participación de las tecnologías es consecuencia del alto costo del Diesel y GN y la política de retiro de Centrales a Carbón; lo cual influye y posibilita la implementación de alta participación ERNC, sumado a la posibilidad de importar electricidad a bajo precio del sistema eléctrico peruano en horas que no cuentan con el recurso solar.

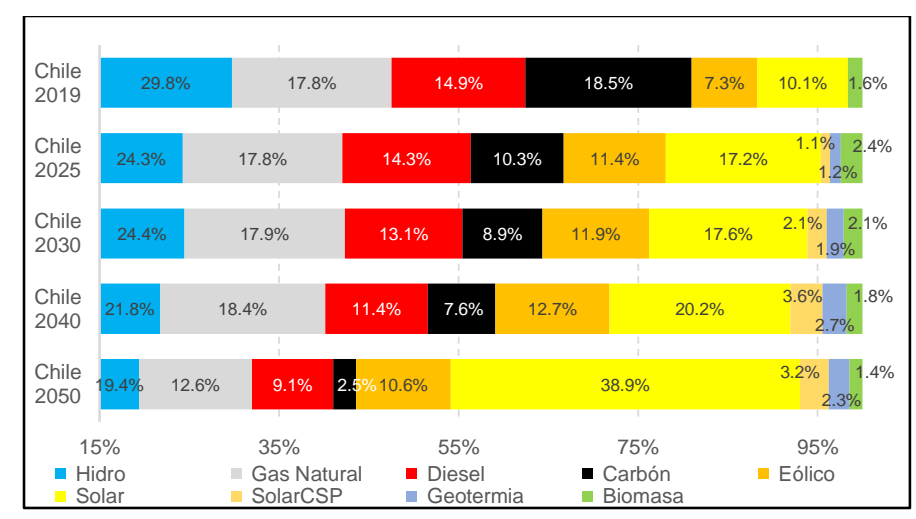

Figura 10: Evolución de la participación por tecnología en Chile para PC01 


\section{Conclusiones}

La diferencia que existe en la disponibilidad de recursos energéticos NR (gas natural) entre Perú y Chile se refleja en las distintas políticas de desarrollo energético de ambos países. Y también es uno de los factores para que exista una mayor diferencia entre sus CMgs, debido a que el Perú posee reservas de GN y cuenta con un subsidio implícito en su precio para generación de electricidad.

La diferencia entre el CMg en Perú y Chile provoca que en todos los escenarios simulados la mayor parte del intercambio de energía se realiza desde Perú a Chile representando entre el $98 \%$ y el $79 \%$ del total de intercambios durante todo el periodo.

En el escenario en que no se implementa el gasoducto surperuano (SIT-Gas), se observa que en el Perú si tiene suficiencia de capacidad de generación eléctrica, por lo que éste no resulta indispensable. Además, los costos marginales se reducirán a mediano y largo plazo.

El cumplimiento de la política «Energía 2050» en Chile, (RER no menor al $70 \%$ ), es confirmada ya que en todos los escenarios y se supera dicho porcentaje. En el scenario simulado donde no se cumple esta meta el CMg en Chile se incrementa considerablemente llegando a presentar picos de hasta 120 US $\$ / M W h$, hacia el final del periodo de análisis.

La política de implementación de centrales CSP en la matriz eléctrica de oferta, hace que el $\mathrm{CMg}$ en Perú y Chile tenga mayor variabilidad llegando a picos de 80 US $\$ / M W h$ y 101 US $\$ / M W h$; respectivamente.
La interconexión Perú - Chile, representa para el sistema eléctrico chileno la posibilidad de disponer de electricidad a bajo costo en horas donde no cuente con recurso solar, con ello reducir su CMg y mejorar la confiabilidad de su sistema.

En el corto y mediano plazo no existe complementariedad energética, solo parcialmente en el largo plazo, cuando el diferencial de CMg se reduce, el intercambio de Perú hacia Chile como mínimo llega al $74 \%$ del total, produciendo un intercambio solo por algunas horas del día cuando se cuenta con alta disponibilidad de recurso solar en Chile.

\section{Agradecimientos}

Los autores brindan un agradecimiento especial al Fondo Nacional de Desarrollo Científico, Tecnológico y de Innovación Tecnológica (Convenio $n{ }^{\circ}$ 207-2015-FONDECYT) por la beca de doctorado en ciencias con mención en energética.

\section{Apéndice A Tabla de Acrónimos}

\begin{tabular}{|l|l|}
\hline BTU & $\begin{array}{l}\text { British Thermal Unit / Unidad } \\
\text { Térmica Británica }\end{array}$ \\
\hline CMg & Costo Marginal \\
\hline GN & Gas Natural \\
\hline MM & Millones \\
\hline MW & Megavatio \\
\hline MWh & Megavatio-hora \\
\hline US\$ & Dólares Norteamericanos \\
\hline VPN & Valor Presente Neto \\
\hline
\end{tabular}

Apéndice B Resultados de Escenarios PC02 PC06 

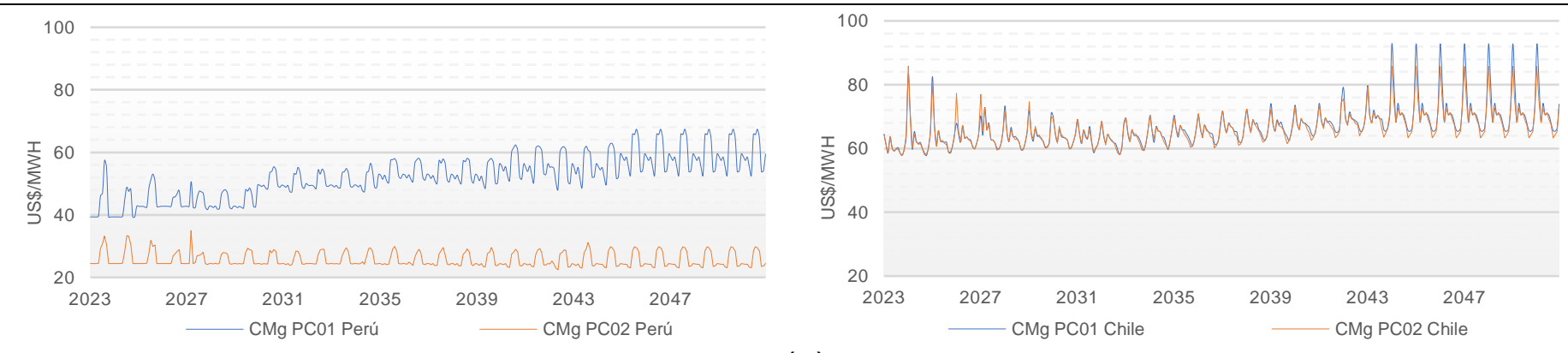

(a)
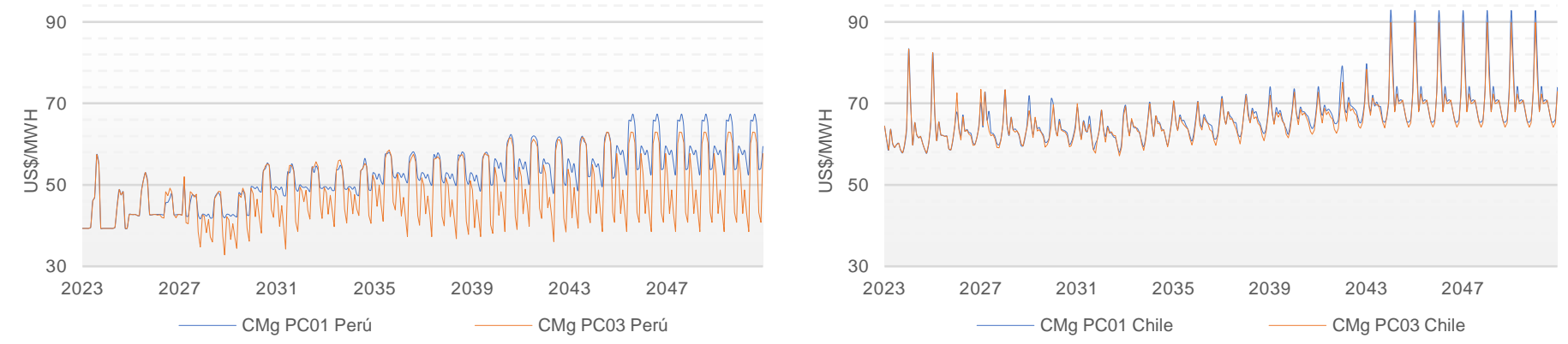

(b)
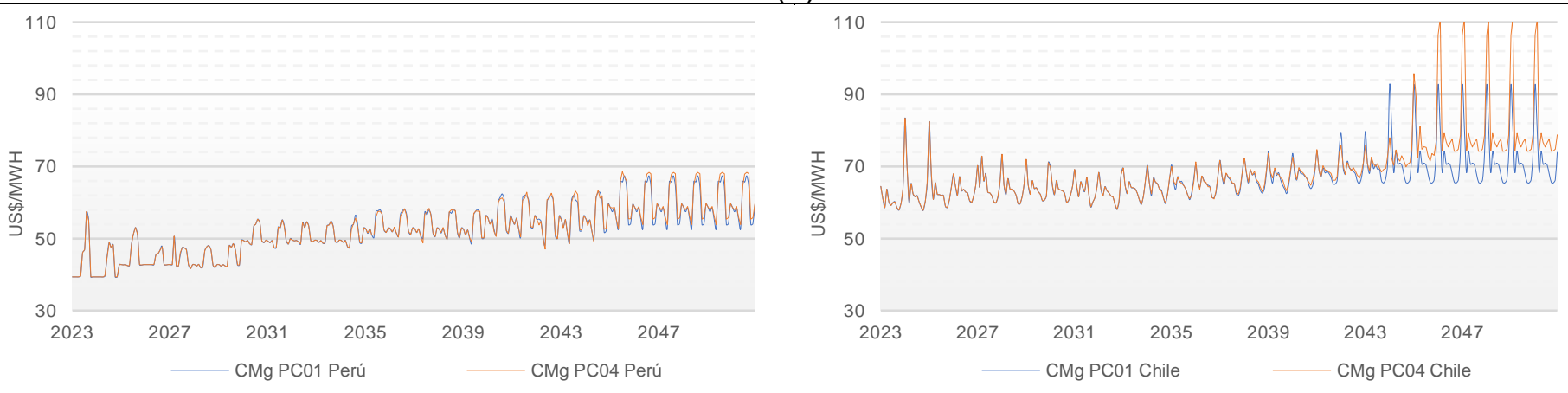

(c)
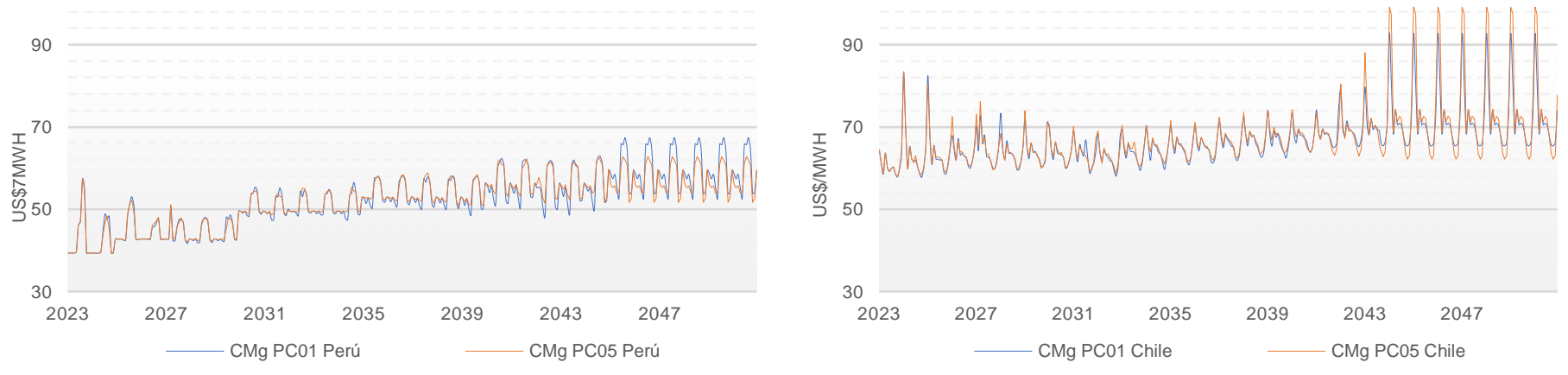

(d)
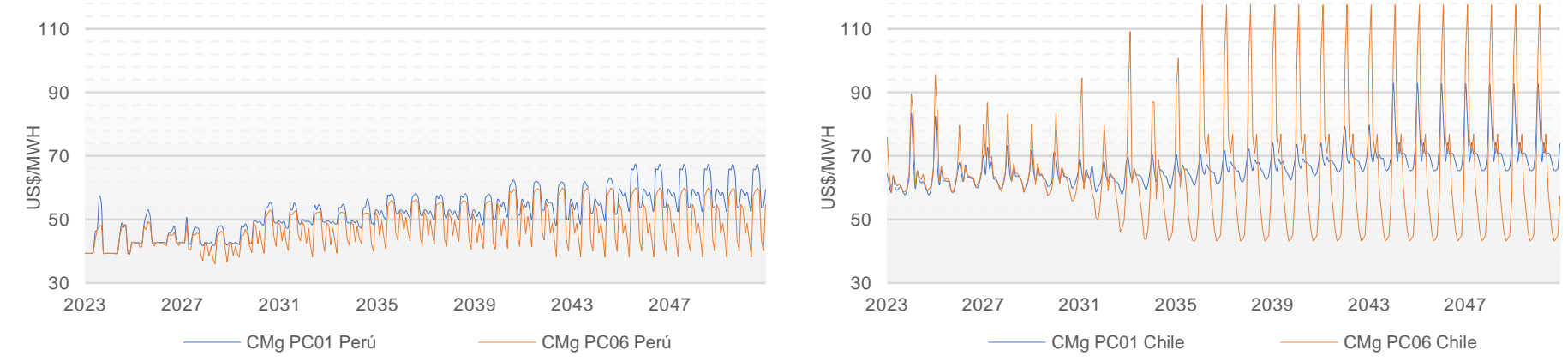

(e)

Figura A1: Variación en los costos marginales de los escenarios Evaluados (US\$/MWh) 

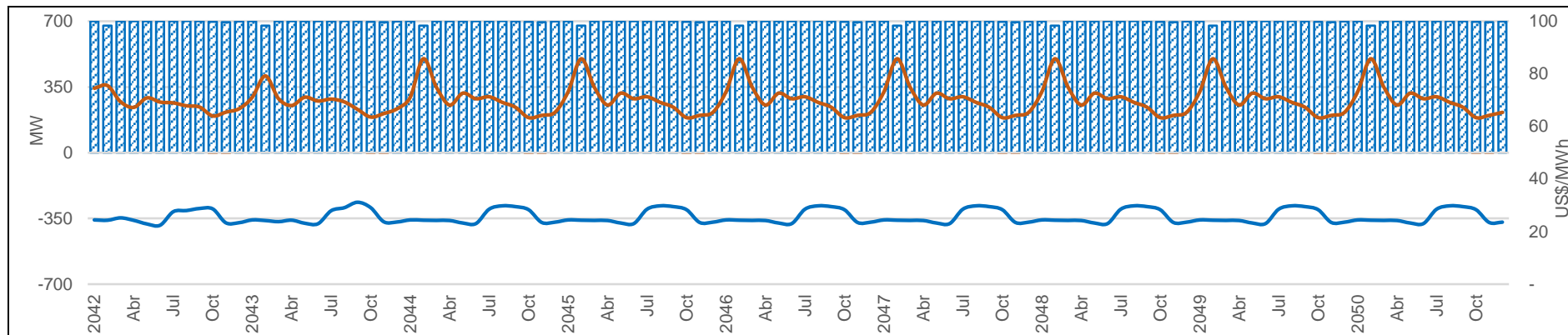

$$
\text { एos Perú - Chile } \quad 000 \text { Chile - Peru }
$$

(a) $\mathrm{CP} 02$

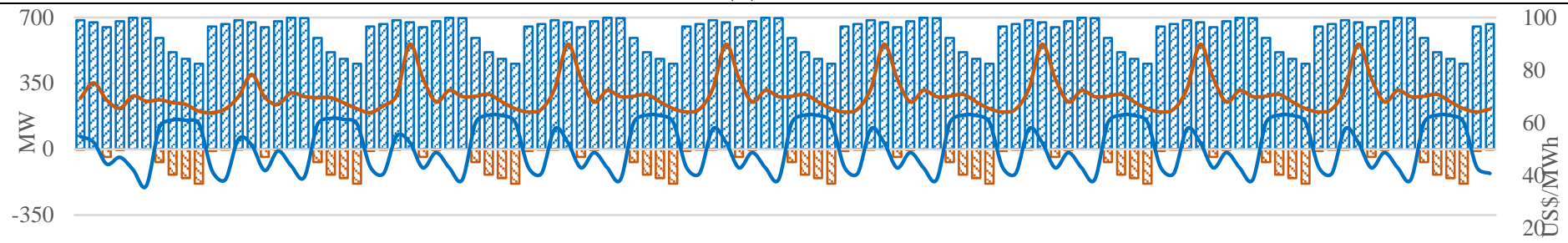

$-700$

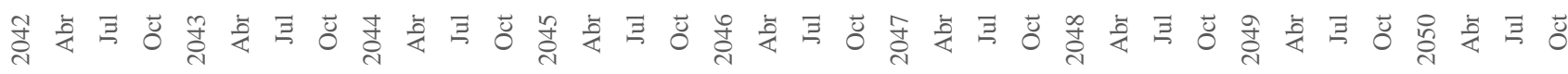

$$
\begin{aligned}
& \text { CKCA Perú - Chile W Chile - Peru }
\end{aligned}
$$

(b) $\mathrm{CP} 03$

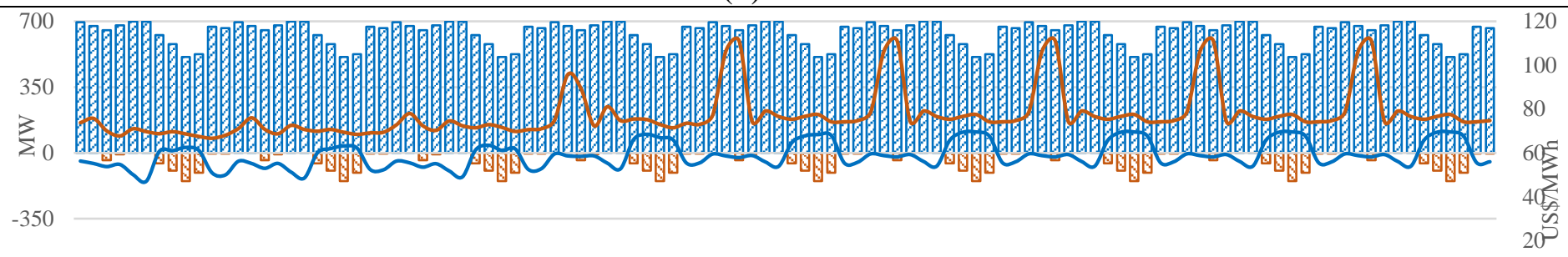

$-700$

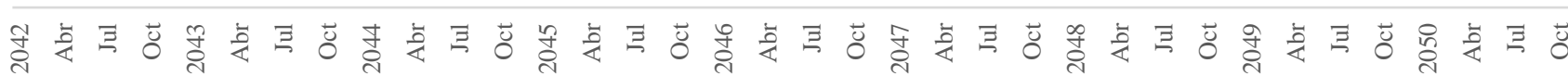

$$
\begin{aligned}
& \text { Æzor Perú - Chile }
\end{aligned}
$$

(c) $\mathrm{CP} 04$

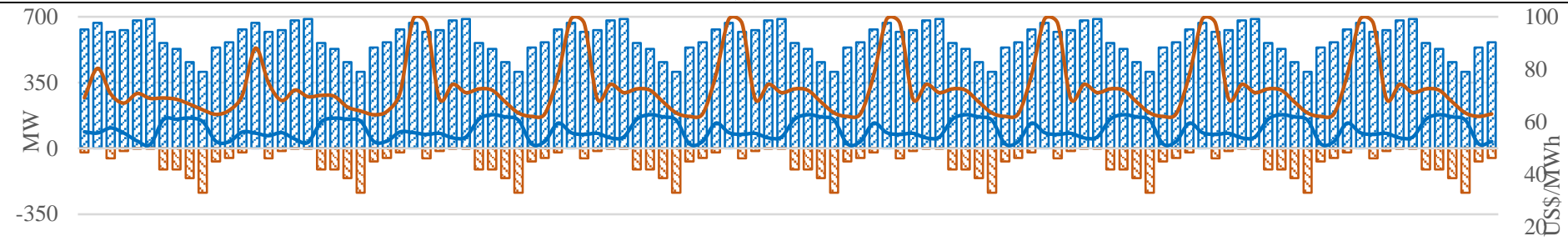

$-700$

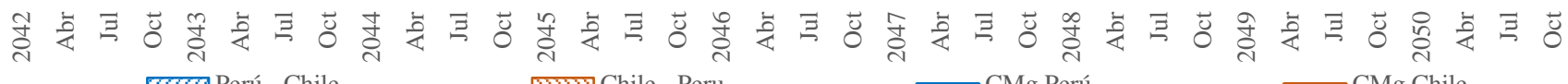

(d) $\mathrm{CP} 05$

Figura A2: Flujo de energía en la interconexión en los escenarios Evaluados

\section{Referencias}

[1] C. Ochoa, I. Dyner y C. J. Franco, "Simulating power integration in Latin America to assess challenges, opportunities, and threats", Energy Policy, vol. 61, p. 267-273, 2013.
[2] R. Poudineh y A. Rubino, "Business model for cross-border interconnections in the Mediterranean basin", Energy Policy, vol. 107, p. 96-108, 2017.

[3] O. Gore y S. Viljainen, "Challenges of crossborder trade between two markets with different designs", de 11th International 
Conference on the European Energy Market (EEM14), 2014.

[4] J. E. Luyo, "El Sector Energía en Perú : Reformas, Crisis, Regulación e Integración Regional", KG, Alemania: Editorial Académica Española- LAP LAMBERT Academic Publishing $\mathrm{GmbH} \&$ Co., abril de 2012

[5] P. Mastropietro, P. Rodilla and C. Batlle, "National capacity mechanisms in the european internal energy market: Opening the doors to neighbours", Energy policy, vol. 28, pp. 38-47, jul 2015.

[6] ] D. Finon, "Capacity Mechanisms and Cross-border Participation: the EU Wide Approach in Question. 2018", HAL, hal01687454, jun 2018.

[7] F. Jara et al., "Interconexión de Mercados Transfronterizos de Electricidad y la Necesidad de Políticas de Coordinación", Revista Tecnia, vol. 28, $\mathrm{n}^{\circ}$ 1, pp. 1-4, dic. 2018.
[8] F. Jara et al., "Expansión a largo plazo de la capacidad de Generación: caso del mercado acoplado Perú-Ecuador", Revista ECIPerú, vol. 17, n² 2, pp. 85-94, dic. 2020.

[9] Ministerio de Energía de Chile, Mesa de Trabajo Descarbonización, [En línea]. Available:

https://www.energia.gob.cl/panel/mesa-detrabajo-descarbonizacion. [Último acceso: 2802 2020]

[10] Comisión Multisectorial para Reforma del Sector Eléctrico, Informe sobre Problemática del Régimen de Declaración de Precios de Gas Natural, Lima, 2019.

[11] ENGIE, Memoria Anual 2019, Lima, 2019

[12] Comisión Nacional de Energía, "Fijación de Precios de Nudo de Corto Plazo", Santiago, 2020

E-mails: fabiolajaraca@gmail.com

jeluyo@yahoo.es

jose.meza.s@uni.pe 